\title{
Medicina tradicional e neurologia na China: reflexões para o sistema de saúde público brasileiro
}

\author{
Traditional medicine and the neurology in China: reflections \\ for the Brazilian public health system
}

\section{Medicina tradicional y neurología en China: reflexiones para el sistema de salud pública brasileño}

\author{
Gislaine Cristina Abe ${ }^{1}$, Paulo Eduardo Ramos², Bruna Terumi Sato \\ Yonamine $^{3}$, Fernando Davino Alves ${ }^{4}$, Tan Tao ${ }^{5}$, Acary Souza Bulle \\ Oliveira ${ }^{6}$
}

\begin{abstract}
1. Neurologista infantil, acupunturista, mestre em Ciências da Saúde pelo Departamento de Neurologia e Neurocirurgia da Universidade Federal de São Paulo. São Paulo-SP, Brasil.

2.Fisioterapeuta, doutor em Ciências da Saúde pelo Departamento de Neurologia e Neurocirurgia da Universidade Federal de São Paulo em parceria com a Tianjin University of Traditional Chinese Medicine, Tianjin, China. São Paulo-SP, Brasil.

3.Fisioterapeuta, especialista em Intervenção Interdisciplinar nas Doenças Neuromusculares pelo Departamento de Neurologia e Neurocirurgia da Universidade Federal de São Paulo. São Paulo-SP, Brasil.

4.Mestre em Ciências da Saúde pela Beijing University of Chinese Medicine em parceria com a China Academy of Chinese Medical Sciences, Beijing, China

5.Doutor em Ciências da Saúde pela Tianjin University of Traditional Chinese Medicine, Tianjin, China.

6.Neurologista, doutor em Neurologia / Neurociências pela Universidade Federal de São Paulo e Pósdoutorado pela Columbia University. São Paulo-SP, Brasil.
\end{abstract}

\begin{abstract}
Resumo
Introdução. A medicina Tradicional chinesa foi incluída no Sistema Único de Saúde brasileiro, e no setor de investigação de doenças neuromusculares da Unifesp, sendo objeto de pesquisas desde 2003. Atualmente, a China integra a medicina ocidental e chinesa, incluindo em neurologia. Objetivo. Descrever o sistema de saúde chinês; a medicina integrativa (medicina tradicional chinesa e ocidental) e a neurologia na China, bem como o processo de formação profissional em medicina. Método. revisão de literatura com as palavras-chave: China, saúde pública, medicina tradicional, medicina chinesa, medicina integrativa, neurologia, em português, espanhol, inglês, nas bases Pubmed, Cochrane, Lilacs, Google scholar, entre 2002-2018. Resultados. Foram selecionados 34 artigos sobre o saúde pública, medicina integrada e processo de formação em medicina, e neurologia na China. A medicina chinesa vem sofrendo grandes modificações, com ocidentalização iniciada em 1917. A medicina integrada na China busca desenvolvimento técnico-científico em universidades, financiada pelo governo. A formação médica, incluindo de neurologistas, está sendo padronizada, seguindo modelos internacionais. Conclusão. A medicina integrada na China tem características especiais, e segue em desenvolvimento. A ocidentalização das pesquisas no pais aumentará o intercâmbio entre o Oriente e Ocidente, ampliando e abrindo perspectivas no campo científico.
\end{abstract}

Unitermos. medicina tradicional chinesa; medicina integrative; China; neurologia; acupuntura

\footnotetext{
Abstract

Introduction. Traditional Chinese Medicine (TCM) was included in the Brazilian Sistema Unificado de Saúde-SUS (Unified Health System), and at the setor de investigação de doenças neuromusculares da Unifesp has been studied since 2003. China has been a part of both Western and Chinese medicine, including its application in neurology. Objective. 1) To place the Chinese health system into a context; 2 ) to outline the evolution of integrated medicine (Western and Chinese medicine) and neurology in China, and 3) to describe the
} 
process of professional education in medicine and particularly in neurology. Method. We conducted a review of the literature based on a search of the databases Pubmed, Cochrane, Lilacs, and Google Scholar for the terms "China", "public health", "traditional medicine", "Chinese medicine", "integrative medicine", and "neurology" in Portuguese, Spanish, and English in the period 2002-2018. Results. We selected 25 articles regarding the Chinese health system, integrated medicine, and concerning the medical education process. Medicine has been undergoing profound changes in China with the Westernization process that began in the 1910s. Integrated medicine seeks technical and scientific development in the universities. Medical education, including that of neurologists, is being standardized in line with international standards. Conclusion. integrative medicine in China is special and and is still under development. The Westernization of research in China will increase the exchange of east and west, open widely possibilities in scientific area.

Keywords. traditional Chinese medicine; integrated medicine; China; neurology; acupuncture

\section{Resumen}

Introducción. En 2003 la medicina tradicional china se ha incluido en el Sistema de Salud Unificado de Brasil - SUS y, desde entonces, ha sido objeto de estudio en el sector de investigación de enfermedades neuromusculares de Unifesp. Actualmente, China integra la medicina occidental y tradicional china, incluida la neurología. Objetivo. describir el sistema de salud chino; medicina integral (medicina tradicional china y occidental) y neurología en China, juntamente con el proceso de educación médica profesional. Método. revisión de la literatura con las palabras clave: China, salud pública, medicina tradicional, medicina china, medicina integrativa, neurología, en portugués, español, inglés, en las bases Pubmed, Cochrane, Lilacs, Google Scholar, desde 2002 hasta 2018. Resultados. Seleccionamos 25 artículos acerca de salud pública, medicina integrada y proceso de capacitación en medicina y neurología en China. La medicina china ha experimentado grandes transformaciones con la occidentalización empezada desde 1917. Financiada por el govierno la medicina integrada en China busca el desarrollo técnico-científico en las universidades. La capacitación médica, incluida la de neurólogos, se estandariza según las normas internacionales. Conclusión. La medicina integradora en China tiene características especiales y sigue su desarrollo. La occidentalización de la investigación científica en China aumentará el intercambio entre Oriente y Occidente, expandiéndose y abriendo nuevas perspectivas en el campo científico.

Palabras clave. medicina tradicional china; medicina integrativa; china; neurología; acupuntura

Trabalho realizado no Setor de Investigação de Doenças Neuromusculares da Universidade Federal de São Paulo - Unifesp, São Paulo-SP, Brasil.

\section{INTRODUÇÃO}

A Organização Mundial de Saúde conceitua medicina tradicional (MT) como o conhecimento baseado em teorias, crenças e experiências de povos indígenas de diferentes culturas, explicáveis ou não pela ciência, usados para a manutenção da saúde, prevenção, diagnose e tratamento 
de doenças físicas e mentais. Abrange tratamento com ervas, partes de animais ou minerais, acupuntura, terapias manuais e terapias espirituais. São técnicas que buscam a integração do ser humano com o meio ambiente e a sociedade. Em 2006 a acupuntura-moxabustão (zhenjiu 針炎), uma parte da Medicina Tradicional Chinesa (MTC), foi incluída oficialmente no Sistema Único de Saúde brasileiro por meio da Política de Práticas Alternativas e Complementares - PNPIC, mas ainda não está integrada ao sistema de tratamento convencional, talvez com exceção na área do tratamento de dor crônica ${ }^{1,2}$.

A Medicina Tradicional Chinesa (MTC) é um sistema médico complexo, que difere da medicina ocidental (MO) na fisiologia, fisiopatologia, diagnóstico e terapias. A MTC é muito utilizada na prevenção de doenças, e sua prática é incentivada pela Organização Mundial de Saúde ${ }^{3,4}$.

Existem críticas em relação à formação dos profissionais em MTC no Ocidente, pois a maioria não tem conhecimento do contexto ambiental e sociocultural da China, aspecto inerente e indissociável do estudo das medicinas tradicionais, repercutindo na maneira como o profissional interpreta as bases teóricas dessa modalidade de tratamento. Como consequência, as técnicas poderiam se tornar atos puramente mecânicos, desvinculadas do contexto inicialmente proposto. Seguindo esse raciocínio, a aplicação da MTC no Brasil também deveria sofrer modificações, seguindo a adequação biológica regional, ambiental e cultural ${ }^{3,5}$. 
A possibilidade de integração das medicinas visa obter mais efetividade no cuidado à saúde. Neste aspecto, há consenso sobre a necessidade de evidências científicas e melhor conhecimento sobre as práticas. Os estudos científicos sobre a integração da medicina ocidental com a MTC têm se multiplicado em todo o mundo e há uma intersecção, pelo menos em parte, de ambas, apesar das racionalidades diferirem em seus fundamentos. Os estudos sobre os mecanismos de ação da acupuntura, do ponto de vista ocidental, principalmente em relação ao mecanismos neurobiológicos da resposta da acupuntura no tratamento da dor, é um dos exemplos mais ilustrativos de pesquisa em medicina integrativa, ou medicina chinesa, neste caso, se referindo a medicina ocidental e medicina tradicional chinesa ${ }^{5,6}$.

As doenças neurológicas têm uma grande atenção na China devido à grande incidência de acidente vascular encefálico no país. Muitas doenças neurológicas são tratadas com acupuntura e fitoterapia, ${ }^{6,7}$. No setor de investigação de doenças neuromusculares da Universidade Federal de São Paulo (Unifesp) a acupuntura foi introduzida em 2003 para fins de pesquisa e atendimento.

A China é o berço da MTC, que traz características socioculturais, ambientais e biológicas, próprias e pertencentes ao seu sistema de diagnóstico e tratamento. Num momento em que se discute a prática da acupuntura e de outras técnicas terapêuticas da MTC no Brasil, consideramos necessário o entendimento de sua história e 
de seu estado atual no contexto em que se insere, com interesse especial no desenvolvimento e atuação na neurologia.

Os objetivos dessa revisão são: 1. Contextualizar o sistema de saúde chinês; 2 . Descrever o processo de formação profissional em medicina e em neurologia na China; 3. Descrever a evolução e o estado atual da medicina tradicional chinesa, da medicina chinesa (associação da medicina ocidental e chinesa) e da neurologia na China.

\section{MÉTODO}

Foi realizada uma revisão de literatura com as palavras chaves: China, saúde pública, medicina tradicional, medicina integrativa e neurologia, em português, espanhol e inglês. A pesquisa foi realizada nas bases Pubmed, Cochrane, Lilacs, e Google scholar, buscando artigos publicados durante o período de 2002 a 2018. Os artigos foram selecionados inicialmente através da leitura do título e resumo. Após identificação do tema principal, foi feita a leitura dos textos completos, com classificação dos assuntos e nova seleção dos artigos em relação aos objetivos desta revisão. 


\section{RESULTADOS}

Foram encontrados 52 artigos relacionados no período de 2002 a 2018. Os artigos foram agrupados em cinco categorias, mediante leitura do título e resumo: 1 . descrição da organização do sistema de saúde público e suas modificações ao longo do tempo; 2. formação dos profissionais; 3 . integração das medicinas chinesa tradicional e ocidental; 4. desigualdade da distribuição dos recursos de saúde (colocando em evidência a desigualdade entre zona urbana $x$ rural e regiões pobres do país); 5 . descrição do financiamento dos recursos destinados e gastos com a saúde. Foram selecionados 34 artigos, concentrados nas categorias um: 13 artigos; dois: 4 artigos; e três:17 artigos, pertinentes ao tema do trabalho. Excluímos as categorias quatro e cinco por não se enquadrarem no escopo do estudo. Textos do tipo "comentários" e textos que se referiam a regiões específicas da China também foram excluídos, devido à grande diversidade regional do país.

\section{DISCUSSÃO}

A China é uma das civilizações mais antigas do mundo e uma das poucas que possuem uma continuidade de sua cultura e história até os dias de hoje, uma das "mais ricas tradições acumuladas pela humanidade" ${ }^{\prime 8,9}$. A medicina tradicional chinesa (MTC) é a única medicina tradicional (MT) inserida atualmente no sistema público de saúde do 
seu país de origem e praticada em todos os níveis de atenção à saúde ao lado da medicina ocidental que conhecemos $^{10}$.

É a MT mais preservada, no sentido de possuir documentação escrita, com extenso material informativo utilizado nas escolas de formação médica tradicional no país e exportado para vários países do Oriente e do Ocidente $^{3}$. Como está inserida no sistema de saúde pública da China, acreditamos que fosse necessário obter as informações de como funciona o sistema como um todo, não subdividido em partes, pois existe uma complexidade que uma simplificação ou segmentação do objeto de estudo poderia alterar a percepção do real significado da presença da MTC até os dias de hoje na China moderna.

Não há como desvincular a MTC da história da China, pois ela está profundamente ligada às características culturais e civilizatória do país, e das mudanças que afetaram o modo como a MTC foi ensinada, praticada e modificada, de acordo com as exigências sociais e culturais ao longo do tempo. As informações referentes ao campo da saúde foram organizadas cronologicamente, correlacionando com os eventos político-sociais contemporâneos que mudaram o cenário da saúde pública na China, para melhor esclarecimento, correlação e interpretação do cenário atual da MTC no país.

O sistema público de saúde chinês tem sido objeto de observação da comunidade mundial. É praticamente um consenso internacional que suas experiências ensinam 0 
que funciona e o que não funciona em termos de sistema de saúde pública, sendo cenário de experiências inovadoras como a dos "médicos pés-descalços", agentes de saúde com formação técnica que atuaram intensamente para suprir a demanda de cuidados médicos durante a década de 70. Sendo um país populoso, responsável por um quinto da população mundial, a prevalência de doenças crônicas cardiovasculares, câncer, doenças infecciosas como a pneumonia asiática, as modificações alimentares e de atividade física, a prevalência de doenças ambientais e comportamentais são uma vitrine para estudo da dinâmica da saúde global ${ }^{(11-18)}$.

A MTC tem origem anterior à invenção da escrita, aproximadamente 2.500 anos a.C. Inicialmente, possuía características mágicas e sobrenaturais, e a figura do médico era associada à figura do xamã. Os medicamentos utilizados eram constituídos por álcool misturado com compostos diversos, pedras arredondadas eram aquecidas para aplicar sobre a pele e pedras com pontas drenavam abcessos $^{3}$.

Na dinastia Zhou (1027-771 a.C.), as concepções mágicas exercidas pelo Xamã começaram a dar origem às bases da medicina como ciência, e surge a figura do médico. As principais teorias da MTC datam deste período, incluindo a teoria do Yin e Yang e dos Cinco Movimentos. Existiam categorias de médicos, estabelecidas pelo imperador, cuja classificação estipulava a gravidade da doença que ele estava apto a tratar. Nessa época, foram 
encontrados os primeiros registros de informações sobre a atuação médica, como os atuais prontuários. Os resultados dos tratamentos eram averiguados, atribuindo-se um índice de cura que cada categoria médica deveria obter, funcionando como um sistema de prestação de contas. Durante a dinastia Han (202 a.C.-248 d.C.), houve maior oposição às crenças supersticiosas e, nesse período, surgiram livros clássicos utilizados até hoje como base teórica da MTC. Sem dúvida, a China possuía uma civilização e, consequentemente, um sistema de medicina muito avançado para a época ${ }^{3}$.

Durante a dinastia Qing (1644-1911), no século XVII, na última linhagem de imperadores chineses, a medicina ocidental começou a ser praticada no país, de modo independente e decorrente do contato entre os chineses e estrangeiros. Até então, em todo o território chinês, a única medicina disponível era a MTC. Somente em 1917 foi inaugurada a primeira universidade de medicina ocidental em Beijing. A China se encontrava numa situação caótica e a medicina convivia com dois paradigmas em relação à doença: a teoria ocidental e a chinesa tradicional. A MTC começou a ser perseguida e foi praticamente banida, livros clássicos foram queimados e os professores exilados. Segundo os historiadores, a medicina tradicional sobreviveu nessa fase devido à perpetuação da tradição oral no interior das províncias ${ }^{3}$.

No século XX, o autor George Soulié de Morant, cônsul da França em Shanghai, começou a divulgar resultados do 
tratamento com acupuntura numa epidemia de cólera. Em 1939 o livro L'Acuponcture Chinoise, uma compilação de textos chineses, foi publicado por Morant e se tornou um marco da divulgação e difusão da aplicação prática da acupuntura no Ocidente ${ }^{3}$.

Em território chinês, a MTC tinha sido proibida até o período da República da China (1949), quando o país estava enfraquecido devido ao período de guerra com 0 Japão. Nessa fase o governo chinês reincorporou a MTC, com ênfase na prevenção de doenças. Os serviços de saúde começaram a ser financiados e dirigidos pelo governo. Havia receio que a MTC fosse substituída pela $M O$, que simbolizava a modernidade e o progresso. As políticas de saúde começaram a ser decididas de forma centralizada e administrada regionalmente pelas províncias. As escolas para a formação de médicos acupunturistas voltaram a funcionar, autorizadas pelo governo da República, e o mesmo reafirmou a importância da MTC, frente a um sentimento nacionalista que tomava conta do país. Citada por vários autores era importante a necessidade de se disponibilizar uma medicina com custos baixos, efetiva e com implantação rápida, para minimizar a situação precária em que se encontrava a população chinesa ${ }^{3,10,18,19}$.

Em 1966, iniciou-se o período conhecido como a "Revolução Cultural" (1966-1969) na China, movimento ideológico político liderado por Mao Tsé-Tung, baseado em argumentos de cunho cultural, mas que tinha motivação fortemente política, devido à pressão da oposição sobre o 
governo em decorrência do desastre do plano econômico anterior, que resultou na morte de milhões de chineses devido à fome $(1958-1961)^{18}$. Houve perseguição às pessoas "intelectualizadas" ou com ideias "pró-ocidentais", opositoras ao governo, e todas as universidades, inclusive as de medicina, foram fechadas por cinco anos. Não havia médicos suficientes para o atendimento da população e a solução foi a implantação da política dos "médicos pésdescalços", técnicos em saúde treinados num período três a seis meses, para atendimento em grande escala na área básica e preventiva da população da zona rural, muitos treinados para executar técnicas simples da MTC, com custos muito baixos. Apesar de ser considerada uma ação inovadora, alguns autores atribuem o caos na saúde que se instalou nos anos subsequentes ao fracasso dessa política de governo, por má administração, falta de planejamento e treinamento inadequado ${ }^{11,18}$.

Na década de 70, o caos na saúde estava instalado. Mao Tse-Tung foi substituído por Deng Xiaoping, que iniciou uma abertura política, mudando radicalmente a postura do governo, visando dar "dignidade e condições de vida aos chineses". As universidades foram reabertas, iniciou-se um período de expansão econômica do país, e as medicinas ocidental e chinesa tradicional começaram a ter um grande desenvolvimento ${ }^{18}$. Em consequência, em 1972, o presidente dos Estados Unidos da América, Richard Nixon, visitou a China. Durante essa visita, um integrante da comitiva norte-americana necessitou de tratamento 
cirúrgico, havendo complicações pós-operatórias tratadas com acupuntura, com resultados amplamente divulgados pela imprensa internacional. Isso marcou o início da popularização da acupuntura no Ocidente, ocorrendo um movimento de interesse crescente no intercâmbio de estudantes para as Faculdades de MTC, e a China passou a receber estudantes de todo o mundo ${ }^{3}$.

Essa fase marcou uma mudança na postura administrativa, com grandes investimentos financeiros em ciência e tecnologia, resultando numa queda na incidência de doenças infecciosas, com diminuição da mortalidade infantil e aumento da longevidade. Milhões de pessoas saíram do nível de pobreza e modificaram o estilo de vida, e surgiram novas questões, como os problemas ambientais, poluição do ar e da água, tornando-se uma ameaça à saúde pública. Houve uma modificação no espectro de doenças, como epidemias de doenças sexualmente transmissíveis, abuso de substâncias químicas, diabetes, obesidade, tabagismo, doenças cardíacas e mentais, relacionadas diretamente ao novo ambiente e comportamento. Aumentaram as doenças crônicas, como doença cardíaca, acidente vascular cerebral, câncer e doenças mentais $^{12,13,18,20}$.

O governo começou a privatizar os serviços de saúde, aumentando os custos dos cuidados em saúde que levaram muitas famílias à falência financeira, e nem todos tinham acesso aos tratamentos. A política do filho único, imposta pelo governo, trouxe a questão do envelhecimento da 
população e o problema 4:2:1 (quatro avós, dois pais e um filho), que sobrecarregava o filho no cuidado com os pais e avós. O cuidado na prevenção às doenças crônicas e a atenção às necessidades do idoso também se tornaram um ponto fundamental para o planejamento das políticas de saúde pública ${ }^{12,13,18,21}$.

Entre as décadas de 80 e 90, o Brasil registrou a visita de várias delegações chinesas interessadas em conhecer a "estratégia brasileira de desenvolvimento nas áreas de telecomunicações, infraestrutura, agricultura, previdência social, saúde e combate à corrupção"22. Isso exemplifica o quanto a China buscava aprender com países que considerava superior ou com potencial para sê-lo.

Pressionado pela população, o governo chinês reviu a questão da privatização e passou a assumir responsabilidade no planejamento e execução dos serviços de saúde. Criou-se subsídio para os serviços, com cobertura parcial dos gastos, e ocorreu a reconstrução dos hospitais com incentivo à formação profissional ${ }^{18}$.

$\mathrm{Na}$ década de 90, constatou-se que o país ainda possuía grandes desafios, havendo grandes desigualdades sociais. Em 1989, ocorreu o episódio de Tiananmen conflito na Praça da Paz Celestial, que evidenciou forte pressão da sociedade, descontente com o governo, com conflito entre conservadores e progressistas. A violência e o autoritarismo do governo chinês, expostos internacionalmente, refletiram em questões comerciais e diplomáticas que forçaram os dirigentes a se posicionarem 
de forma diferente em diversas áreas, como a reivindicação da garantia ao acesso aos serviços de saúde e, novamente, o cuidado com a qualidade de vida da população. Com isso, Deng Xiaoping declarou novos grandes investimentos na área de saúde, ciência e tecnologia ${ }^{18}$.

Houve uma melhora na oferta de serviços de saúde, porém em 2003 ocorreu a epidemia de pneumonia asiática (Severe acute respiratory syndrome - SARS), provocando grande número de internações e mortes devido à gravidade da doença. Apesar dos esforços investidos, esse incidente mostrou várias deficiências no sistema de saúde e uma nova crise política se instalou devido à grande insatisfação popular ${ }^{18}$.

O governo chinês foi obrigado a anunciar novas mudanças em relação à saúde pública, com maior investimento financeiro e com o incentivo maciço da área da saúde e da educação, modernizando hospitais, promovendo o acesso aos recursos, formando profissionais, incentivando a medicina integrada e aumentando a cobertura de riscos. A participação da China em instituições internacionais vem mudando a forma como o país assume responsabilidades globais, incluindo os setores sociais e de saúde, através da OMS ${ }^{12,14,17,23,24}$.

Em decorrência desses esforços, a China vem demonstrando uma melhora nos indicadores de saúde. Mas a taxa de mortalidade, que era de 6.9/1000 em 2004, em 2017 atingiu de 7,8/1000, taxa elevada principalmente devido a desastres naturais, como terremotos e tufões, e 
epidemias de doenças infecciosas, como tuberculose, tifo, esquistossomose ou dengue ${ }^{19,25}$.

A grande extensão do território chinês, assim como no Brasil, faz com que existam diferenças regionais marcantes, que afetam a maneira como recursos de atenção à saúde são oferecidos. Essas diferenças, principalmente entre os grandes centros urbanos e a área rural, é um dos inúmeros desafios a serem superados pelo governo chinês, que encara o financiamento das ações como sendo fundamental para a oferta de um serviço de qualidade. O país, atualmente considerado socialista de mercado, mas com características peculiares chinesas, optou por receber orientações para todas as modificações no planejamento da saúde de instituições externas, que foram estudadas e propostas por grupos de especialistas, incluindo o Banco Mundial e a Organização Mundial de Saúde $\mathrm{e}^{11,13,14,22-24}$.

A estrutura dos cuidados de saúde está hierarquizada nas áreas urbanas e rurais, e baseia-se em três camadas. Nas áreas rurais, uma rede de unidades básicas fornece serviços primários de saúde em quase todas as aldeias, operados por vários funcionários em tempo parcial. As clínicas também organizam campanhas de saúde pública, incluindo os programas de imunização. Existe uma dificuldade na fixação dos profissionais da saúde nesses centros devido aos baixos salários, condições de moradia e menor oferta de oportunidades para desenvolvimento profissional $^{12,19}$. 
Em seguida na hierarquia são 37.128 centros de saúde, que são unidades maiores, com atendimento médico em tempo integral, com a prestação de cuidados de saúde primários e supervisão dos serviços médicos prestados pelas clínicas de saúde pública ${ }^{25-27}$.

Nas áreas urbanas, o nível básico de prestação de cuidados de saúde é semelhante ao da zona rural. Em seguida na hierarquia, são os Hospitais Distritais que prestam cuidados de saúde primários e organização administrativa. A camada final na hierarquia é o Hospital Municipal/Provincial, que oferece atendimento especializado e de referência. O sistema de saúde hierarquizado é, pelo menos em teoria, projetado para fornecer acesso equitativo e acessível aos cuidados de saúde de qualidade para todos os membros da sociedade chinesa. Mas apesar de todos esses esforços, a China ainda é citada como um dos países de maior desigualdade no acesso à saúde.

\section{Formação em Medicina tradicional chinesa (MTC)}

Antigamente, na China Imperial, a formação em medicina tradicional chinesa era feita por quatro escolas tradicionais: 1) Escola dos religiosos: conhecimento e treinamento transmitido dentro de templos ou sociedades (Taoísmo, Confucionismo, Legalismo, Maoísmo, Budismo) aos seus iniciados; 2) Escola familiar: conhecimento e treinamento passado de gerações a gerações, dentro de uma família, normalmente, aos filhos do sexo masculino; 3) Escola dos artistas marciais: conhecimento e treinamento 
transmitido dentro das escolas militares, conhecimento utilizado para socorrer feridos em batalhas e estimular 0 autocuidado durante períodos de paz, e/ou a artistas marciais que utilizavam as informações para bloqueios de pontos que interferem na circulação de energia e sangue durante confrontos marciais; 4) Escola dos médicos e/ou acadêmicos: transmitido pelo ministro da saúde imperial em curso elementar a seus discípulos ${ }^{10,28}$.

A transmissão do conhecimento segue modelo estruturado com base no modelo das escolas médicas do Ocidente, pela influência e contato com médicos ocidentais com o advento da república e da queda do império. A legislação chinesa de 1998 regulamentou o exercício da MTC e acupuntura na rede de saúde para dois tipos de formação: 1) profissionais graduados em medicina tradicional chinesa e/ou acupuntura como bacharelado, acrescido de três anos de residência; 2) profissionais de linhagem familiar e/ou mestre/discípulo, com experiência clínica superior a três anos. O mestre e/ou líder familiar deve estar filiado a uma associação tradicional e indica seu discípulo, quando apto ao exercício, para filiação. Em ambos os casos, é necessário realizar o exame nacional para adquirir o registro e autorização para exercício profissional. As demais escolas de ensino podem atuar nos templos, espaço de ensino das artes marciais e/ou consultórios, contudo, a lei não permite que atuem em hospitais ou na rede de saúde ${ }^{10,28}$. 
Atualmente, nas universidades, quase não se encontra - modelo de MTC exclusiva. Na maior parte das universidades, a grade curricular apresenta $70 \%$ de MTC e $30 \%$ de MO, em virtude do esforço do governo em desenvolver a medicina integrativa chinesa (medicina tradicional integrada com a medicina ocidental). Nas clínicas de família, $17,4 \%$ dos médicos têm formação exclusiva de MTC, 32,3\% em MI; e 50,3\% em MO. Existem $13,8 \%$ de hospitais exclusivos de MTC no país ${ }^{10,26,29-32}$.

\section{Formação em Medicina ocidental (MO)}

A primeira turma de medicina ocidental baseada em evidências foi criada em 1917, em Beijing, na universidade chamada Peking Union Medical College, construída pela fundação Rockefeller. Na época da Revolução Cultural, com a extinção das universidades e da formação universitária em medicina, os professores foram exilados. As universidades reabriram somente em 1970 e, durante esse período, houve uma grande escassez de profissionais e uma piora considerável na oferta de serviços em saúde ${ }^{33}$.

Atualmente, a China possui uma grande diversidade de programas para formação médica, com duração de três a oito anos de estudo. A palavra "médico" é traduzida como "Yisheng" (醫生). Esse termo designa vários tipos de formação na área da saúde. Isso causa uma grande confusão, pois não há como saber, através da tradução "médico", qual a formação do profissional se tomarmos como base o modelo ocidental. O curso de cinco anos 
garante o título de bacharel, o de sete anos corresponde ao mestrado, e, o de oito, doutorado. O curso de três anos confere o título de médico assistente. Além disso, o médico também pode ter formação em faculdade, ou em escola de segundo grau ou técnica. O governo se esforça para formar profissionais com alto grau de escolaridade, bacharéis ou com mestrado e doutorado, visando obter maior qualidade no atendimento da população 23,33-35.

\section{Neurologia}

A neurologia na China começou a existir como entidade independente em meados de 1920, introduzida por um neurologista americano, Andrew Woods, na Peking Union Medical College. Outros médicos, formados em neurologia e neurocirurgia na Europa, Estados Unidos da América, Canadá, voltaram à China e iniciaram a prática e treinamento de novos alunos na especialidade em Beijing (Pequim), Tianjin, Shanghai, Changsha e Nanjing. Houve forte influência da Rússia no pós-guerra, na década de 50. Em 1952, a Associação Chinesa de Neurologia e Psiquiatria foi estabelecida, e, em 1955, o Jornal de Neurologia e Psiquiatria chinês foi publicado, havendo uma grande expansão da neurologia no país ${ }^{36}$.

No período da Revolução Cultural, em 1966, também houve um grande retrocesso da especialidade. Houve a incorporação da neurologia pela medicina interna, com a marginalização da especialidade e dos neurologistas. A publicação de seu único periódico acadêmico foi suspensa, 
todas as atividades acadêmicas foram canceladas e o desenvolvimento de neurologia praticamente cessou ${ }^{36}$.

O cenário socioeconômico começou a se modificar a partir de 1977, com o crescimento econômico da China. Houve um aumento considerável no Produto Interno Bruto e a economia teve uma expansão muito rápida, com impacto em questões relacionadas à saúde. Os exames complementares, tais como eletroencefalograma, tomografia computadorizada, ressonância magnética, foram disponibilizados em grandes hospitais, melhorando o sistema de diagnóstico. Ocorreu uma expansão rápida da neurologia nessa fase, talvez pela percepção dos médicos generalistas e pelos próprios pacientes da complexidade das doenças neurológicas e necessidade do acompanhamento por um especialista. Atualmente, 0 atendimento em neurologia é concentrado em grandes hospitais. Um dos maiores centros de neurologia da China, Beijing Xuan Wu Hospital, registrou 24,6 milhões consultas entre 2001 a 2006. Em 2011, as consultas excederam $1.300 / \mathrm{dia}^{36}$.

Para a especialização em neurologia, são necessários mais 3 a 4 anos após a graduação em medicina, que dura 5 anos. O doutorado em neurologia dura 3 anos, com ênfase em clínica ou pesquisa. A subespecialização é um campo recente, mas existe treinamento em atendimento neuropediátrico, reabilitação e doenças vasculares, principalmente em acidente vascular encefálico. A demanda 
por serviços mais especializados tem aumentado e é uma tendência para o aprimoramento dos serviços de saúde ${ }^{36}$.

A ciência e tecnologia são incentivadas, visando principalmente à produção de novos produtos. A pesquisa translacional com células-tronco, para doenças como esclerose lateral amiotrófica, paralisia cerebral e esclerose múltipla, é objeto de muito interesse por parte dos neurologistas e neurocirurgiões chineses. A falta de transparência na publicação das pesquisas e o tipo de modelo experimental, não aceito no Ocidente, fez com que em 2009 o Ministério da Saúde da China normatizasse esse tipo de pesquisa. Assim, instituições que desejam oferecer terapias com células-tronco deverão, primeiro, demonstrar a segurança e eficácia em ensaios clínicos multicêntricos, duplo-cego e randomizados. Em seguida, o tratamento será avaliado por um departamento regulador do Ministério da Saúde, estando disponível somente após essa validação ${ }^{36}$.

\section{Medicina chinesa (medicina ocidental integrada com a medicina tradicional chinesa)}

Historicamente, a prática da medicina tradicional na China retornou em 1949, pois estava abolida pelo governo. A medicina ocidental se espalhou pelo país, levando a ideia de modernidade e avanço tecnológico, e as práticas tradicionais foram associadas ao passado e ao atraso tecnológico. Muitas razões são apontadas para que o governo se interessasse pelo retorno das práticas tradicionais de medicina. Além da motivação política, com o 
argumento nacionalista, outro provável motivo é o econômico, pois o país estava em crise econômica e as terapias chinesas tinham um custo infinitamente menor. Em 1949, Mao Zedong promoveu o primeiro Congresso Nacional para administração da saúde pública, oficializando a união entre a medicina tradicional e a medicina ocidental, criando a medicina chinesa (MC). Essa foi a base para a atuação dos médicos pés-descalços ${ }^{30,32}$.

A MO já era praticada por todo o território chinês, devido ao contato com o Ocidente, mas a formação para médico de família ou clínico geral, de maneira oficial, começou na China na década de 80, após a abertura política. A MTC é incluída no currículo, também com carga horária de 3 meses. A formação ambulatorial inclui medicina preventiva, cuidados em saúde, reabilitação física e planejamento familiar, em estágios de 6 meses de duração em cada setor 26,31-34.

Segundo o Mao Zedong o "futuro e a solução dos problemas viriam com a integração das duas medicinas". Nos anos seguintes, houve uma grande expansão da MO. A associação com a imagem de progresso e modernidade fez com que a população chinesa também tivesse preferência pelo tratamento ocidental. Antagonicamente, em 1980, foi verificado o estado de decadência dos hospitais de MTC e o governo chinês teve que se comprometer a incentivar a prática da MC, passando a ser o responsável pela regulamentação na República Popular da China, destinando 
grandes recursos financeiros para a reforma do sistema de saúde ${ }^{18}$.

Nesse sentido, somente em 2003 foi publicado um documento com as regulações propostas para 0 desenvolvimento da MC, com suporte e proteção, e o incentivo para que os profissionais da área médica se empenhassem para "aprender e cooperar uns com os outros". Lembramos que, nesse período, houve a epidemia da pneumonia asiática (SARS) e, em muitos hospitais, o tratamento foi efetuado com MO e MTC em associação, fato que impulsionou esse posicionamento em relação às pesquisas com MC. Em 2006, foi criada uma normativa para o planejamento e execução de atividades com 0 comprometimento de dezesseis ministros com a modernização da $\mathrm{MTC}^{10,26,29,31,32}$.

A prática de MTC na China nos dias atuais é considerada prática de $\mathrm{MC}$, e, em virtude disso, se propôs a nomeação do termo "medicina chinesa", substituindo a antiga denominação "MTC". Existem cerca de 300 hospitais de MC no país. Nos hospitais de MO, existem departamentos de MTC em 95\% das instituições. Cerca de $40 \%$ dos cuidados em saúde na China são baseados na MTC, principalmente na atenção básica em ações preventivas $^{10,29}$.

Existem cerca de cem institutos de pesquisa independentes cadastrados, atuando nessa área. A pesquisa básica inclui o estudo do pulso e da língua, com modernos aparelhos de avaliação de imagem da língua, 
captação do tipo de pulso e reprodução dos pulsos para o estudo dos alunos, controlados por computador. Um dos maiores centros de pesquisa é o "China Academy of TCM" em Beijing, que possui 60 anos de fundação, com 4000 membros associados, e atua como colaborador da Organização Mundial de Saúde no departamento de medicinas tradicionais, que existe desde $1972^{26,37}$.

Com a SARS houve uma nova disposição no fortalecimento da integração da MTC com a medicina ocidental, devido aos resultados positivos, após a associação de ambas no tratamento dos doentes, com uma redução drástica na mortalidade. Em Beijing, essa redução atingiu $80 \%$. A taxa de mortalidade mundial foi de 9,5\%, enquanto na China foi de $6,5 \%$ e, especificamente em Beijing, 4\% $26,29,30,38$.

Resultados promissores de pesquisa clínica com abdome agudo, cirurgias de revascularização miocárdica, hepatite/fibrose/cirrose hepática, pancreatite, diabetes mellitus, vitiligo e síndrome de estase sanguínea vêm sendo divulgados em publicações cientificas. Houve redução do índice de cirurgia para abdome agudo em casos de pancreatite aguda, colangite aguda e cálculos biliares complicados. O princípio de tratamento utilizado foi o uso de ervas purgativas com influência na transmissão de informações do sistema imunológico, afetando a rede neuroimunoendócrina envolvida nessas situações clinicas ${ }^{38}$.

Uma das teorias da MTC é a de tratar doenças de intoxicação com "veneno" em preparações modificadas, 
uma frase que descreve "o modo chinês de tratar o câncer", que envolve o tratamento com MC. As características das síndromes e doenças estão sendo reconhecidas e aprofundadas através da combinação da cognição moderna da patologia, diagnóstico e estudo de farmacologia em $\mathrm{MO}^{38,39}$.

Existem muitas críticas em relação à qualidade dos estudos científicos e as publicações feitas em chinês. Devido ao aumento do uso das medicinas alternativas e complementares (MACs) pela população de países desenvolvidos e ao uso muito frequente da MT dos países na África (80\%), vem à tona uma ampla discussão a respeito da integralidade e acessibilidade à saúde, que esbarra na necessidade de evidências cientificas visando diminuir "comportamentos impróprios, como lucro desmedido, marketing enganoso, manipulações inescrupulosas", favorecidos pela ausência de regulação, controle de segurança, registro de eficácia e qualificação de competências. A ocidentalização ou homogeneização das MACs é difícil e necessita de atenção, com o risco de se gerar "aumento de custos, perda de identidade, desenraizamento cultural, confusão e perda de função social"130,40-42. A insuficiência de evidências no manejo clínico, ao controle de qualidade e na segurança são fatores limitantes na integração da MTC e $\mathrm{MO}^{40}$.

Na China, também existem críticas em relação ao modo como as pesquisas em MC vêm sendo conduzidas. Alguns autores acreditam que não há como estudar os 
resultados se as pesquisas adotarem o método ocidental para observar os fenômenos, descaracterizando a MTC. Assim, o pensamento da medicina tradicional seria influenciado e, consequentemente, o modo como vemos e analisamos os resultados, com perda de identidade, qualidade e profundidade $\mathrm{e}^{43,44}$.

Outra questão é a dupla prescrição, que ocorre com frequência com os médicos que praticam MC na China, que utilizam medicamentos da MO, combinados ou associados aos da MTC, baseados em experiência clínica pessoal, sem saber ao certo o efeito da associação de terapias, que pode ser sinérgico ou não, podendo haver toxicidade ou sobreposição de efeitos. Uma das iniciativas para reduzir esse problema é a criação de normativas para o tratamento de determinadas doenças, baseada em pesquisas científicas, que visa diminuir custos e oferecer segurança aos médicos e usuários, padronizando tratamentos da MI. Atualmente, existem normatizações clínicas para SARS, úlcera péptica, gastrite, fibrose hepática, câncer de pequenas células do pulmão, doença cerebrovascular e doença coronariana $38,43,44$.

Em países como a Coreia e o Japão, a MT seguiu rumos diferentes, provavelmente devido à influência histórica, cultural e social desses países, embora tivessem origem na medicina chinesa. Assim, pode-se considerar que a MI possa ter diferentes caminhos, dependendo dos fatores culturais e históricos da região em que é praticada. Assim como o Japão, a Coreia também já inicia sua 
produção de protocolos de tratamento, baseada em pesquisas científicas ${ }^{31,32}$.

Segundo a OMS, quatro sistemas médicos tradicionais principais puderam ser identificados: 1 . Medicina tradicional chinesa - China, Coreia e Japão; 2. Ayurvedica - Índia; 3. Unani - países árabes; 4. Tibetana - Tibet. Porém, o único sistema que existe atualmente integrado com a medicina ocidental é a MTC. No Brasil, a medicina tradicional autêntica seria a indígena, e as MACs seriam práticas diversas incorporadas, incluindo a MTC $26,30,31,39,40,42$.

Uma grande utilização das MTs é entre os "médicos de família" chineses, que buscam atender a demanda primária não hospitalar e atendem em centros comunitários de saúde. Nessas unidades, que se espalham regionalmente, 0 médico e a equipe de enfermagem fazem 0 acompanhamento local, com visitas à residência, palestras em saúde, desenvolvimento de material visual, dando ênfase ao trabalho preventivo, levando em consideração o envelhecimento da população e a mudança do perfil de doenças, semelhantes ao Programa Saúde da Família brasileiro. A MTC tem uma grande representação nesse setor $^{29,32,34,45}$.

A fitoterapia tradicional chinesa é outra grande área de pesquisa da MC. Fonte milenar de tratamento das diversas doenças na China é de onde se espera que se isolem as estruturas bioquímicas, oferecendo novos compostos farmacêuticos para tratamento de doenças neurológicas. Muitos estudos têm se concentrado nessa 
área, devido à alta incidência de acidente vascular encefálico na população $26,36,46,47$.

Um hospital com grande experiência em neurologia e medicina integrada é o Hospital da universidade de Medicina Tradicional Chinesa de Tianjin (TUTCM). Este hospital é famoso devido a uma técnica de acupuntura desenvolvida inicialmente para tratamento de acidente vascular encefálico, mas atualmente aplicada em outras doenças neurológicas, denominada XingNaoKaiQiao (醒腦開 憿 $)^{46}$.

$\mathrm{Na}$ China, a população tem livre escolha em relação ao tipo de terapia que deseja receber. Embora haja preferência da população chinesa pelo uso da medicina ocidental nos casos agudos, nos casos crônicos, os usuários relatam preferir o tratamento com MTC. Existe também a diferença em relação aos baixos custos da MTC em relação à medicina ocidental ${ }^{32}$.

A adaptação às necessidades regionais é uma das características da medicina integrada na China, porque a MTC leva em consideração aspectos individuais e coletivos, como as características relacionadas à região. Ainda não há um modelo fixo para o ensino da medicina integrada, mas um autor cita a necessidade de grande criatividade para os alunos praticarem a associação de ambas as medicinas ${ }^{32,48}$. 


\section{CONCLUSÃO}

A China é um país com uma das mais antigas e contínuas civilizações que conhecemos, e mantém uma preocupação com a busca contínua de novas informações que contribuam para o planejamento e execução de sua estratégia de desenvolvimento, inclusive aprendendo com experiências do Brasil. Apesar de geograficamente distante, existem muitas semelhanças entre os dois países, incluindo dimensões territoriais e diversidade de recursos naturais.

A formação médica na China, incluindo de neurologistas, é padronizada, seguindo padrões internacionais. Um dos interesses do governo chinês é melhorar a capacitação dos profissionais da área de saúde, ampliando a oferta dos cursos de pós-graduação, incluindo mestrado e doutorado em medicina, e formação de especialistas. A importação/exportação de especialistas e pesquisadores é uma realidade facilmente identificada nas publicações científicas mundiais.

A China absorveu o modelo ocidental de medicina e de pesquisa e, atualmente, começa a delinear o modelo chinês de medicina integrativa, buscando melhores resultados em vários aspectos, inclusive o financeiro. Esta experiência pode auxiliar no direcionamento dos estudos e pesquisas no Ocidente, visando atingir a integralidade na assistência à saúde, ampliando e abrindo perspectivas no campo de conhecimento da medicina. 


\section{AGRADECIMENTOS}

À Secretaria de Estado de Saúde de São Paulo e CAPES. Especialmente para Rodrigo Arêas Ribeiro de Castro e Manuela Abe Arêas Ribeiro de Castro; carinhosamente para Wu Pi Chun (Evelyn Wu), Nelson Guenka, Mitsue Abe Guenka, que possibilitaram a realização desse trabalho. Correção e revisão ortográfica de Márcia Souza Bulle Oliveira e Eneida de Souza Bulle Oliveira (in memoriam).

Bolsista da CAPES, Proc. No.11/06/2332. Coordenação de Aperfeiçoamento de Pessoal de Nível Superior SBN, Quadra 02, lote 06, Bloco L, CEP 70040-020. Brasília-DF, Brasil.

\section{REFERÊNCIAS}

1.Política Nacional de Práticas Integrativas e Complementares no SUS - PNPIC no SUS (Endereço na Internet). Brasil: Ministério da Saúde; 2006 (acessado 2018). Dsiponível em: http://dab.saude.gov.br/docs/publicacoes/geral/pnpic.pdf 2.PORTARIA No 971, DE 3 DE MAIO DE 2006 - Aprova a Política Nacional de Práticas Integrativas e Complementares (PNPIC) no Sistema Único de Saúde (Endereço na Internet). Brasil: Ministério da Saúde; 2006. (acessado 2018). Dsiponível em: http://portal.saude.gov.br/portal/arquivos/pdf/PNPIC.pdf

3.Carneiro NM. Fundamentos da acupuntura médica. Florianópolis: Editora Sistema; 2001, 147p.

4.Abe GC, Ramos PE, Fontes SV, Yonamine BTS, Mello CAdS, Quadros $A A J$, et al. Poliomielite e síndrome pós-polio pela medicina tradicional chinesa: da fisiopatologia ao diagnóstico. Rev Neurocienc 2011;19:365-81.

http://www.revistaneurociencias.com.br/edicoes/2011/RN1902/revis ao\%2019\%2002/471\%20revisao.pdf

5.Luz MT. Medicina e racionalidades médicas: estudo comparativo da medicina ocidental contemporânea, homeopática, chinesa e ayurvédica. In: Canesqui AM (ed.). Ciências sociais e saúde para o ensino médico. São Paulo: Hucitec/FAPESP; 2000, p.181-200. 
6.Dong J. The relationship between Traditional Chinese Medicine and Modern Medicine. J Evid Based Complementary Altern Med 2013:110. http://dx.doi.org/10.1155/2013/153148

7.Fong G, Cheng T, Lam K, Cheng W, Mok K, Cheung C, et al. An epidemiological study of motor neuron disease in Hong Kong. Amyotroph Lateral Scler Other Motor Neuron Disord 2005;6:164-8. http://dx.doi.org/10.1080/14660820510028412a

8.China CB (endereço na internet). São Paulo: CCIBC, 2015. (acessado em 2018). Disponível em: www.camarabrasilchina.com.br 9.Ponte Y. Uma perspectiva chinesa sobre os fatores culturais a serem considerados pelo brasileiro no processo de negociação com o chinês. [Dissertação de mestrado]. São Paulo: Fundação Getúlio Vargas; 2007.

10. Hesketh T, Zhu WX. Health in China. Traditional Chinese Medicine: one country, two systems. BMJ 1997;315:115-7.

http://dx.doi.org/10.1136/bmj.315.7100.115

11.Zhang X, Kanbur R. Spatial inequality in education and health care in China. China Economic Rev 2005;16:189-204.

http://dx.doi.org/10.1016/j.chieco.2005.02.002

12.Meng Q, Xu L, Zhang Y, Qian J, Cai M, Xin Y, et al. Trends in access to health services and financial protection in China between 2003 and 2011: a cross-sectional study. Lancet 2012;379:805-14. http://dx.doi.org/10.1016/S0140-6736(12)60278-5

13.Ramesh $\mathrm{M}, \mathrm{Wu} X$. Health policy reform in China: lessons from Asia. Soc Sci Med 2009;68:2256-62.

http://dx.doi.org/10.1016/j.socscimed.2009.03.038

14.Tang S, Meng Q, Chen L, Bekedam H, Evans T, Whitehead M. Tackling the challenges to health equity in China. Lancet 2008;372:1493-501.

http://dx.doi.org/10.1016/S0140-

6736(08)61364-1

15.Wang H. A dilemma of Chinese healthcare reform: How to redefine government roles? China Economic Rev 2009;20:598-604. https://doi.org/10.1016/j.chieco.2009.04.001

16.Yang Y, Yang D. Community health service centers in China, not always trusted by the populations they serve? China Economic Rev 2009;20:620-4. https://doi.org/10.1016/j.chieco.2009.04.004

17. Han Q, Chen L, Evans T, Horton R. China and global health. Lancet 2008;372:1439-41. https://doi.org/10.1016/S0140-6736(08)61350-1 18.Dong Z, Phillips M. Evolution of China's health-care system. Lancet 2008;372:1715-6. https://doi.org/10.1016/S0140-6736(08)61351-3

19.Drummer T, Cook I. Health in China and India: A cross-country comparison in a context of rapid globalisation. Soc Sci Med 2008;67:590-605. https://doi.org/10.1016/j.socscimed.2008.04.019 20.Ma J, Lu M, Quan H. From a national, centrally planned health system to a system based on the market: lessons from China. Health Aff 2008;27:937-48. https://doi.org/10.1377/hlthaff.27.4.937

21.Yang G, Kong L, Zhao W, Wan X, Zhai Y, Chen L, et al. Emergence of chronic non-communicable diseases in China. Lancet 
$\underline{5}$

22.Biato Junior O. A parceria estratégica sino-brasileira: origens, evolução e perspectivas (1993-2006). Brasília: FUNAG; 2010, 440p.

23. Anand S, Fan V, Zhang J, Zhang L, Ke Y, Dong Z, et al. China's human resources for health: quantity, quality, and distribution. Lancet 2008;372:1774-81. https://doi.org/10.1016/S01406736(08)61363-X

24.Zhang J, Yan F, Tang S. The incoherence of China's national health development strategies. Am J Public Health 2012;102:14-6. https://doi.org/10.2105/AJPH.2012.301046

25. National Bureau of Statistics of China: National Bureau of Statiscs of China (endereço na internet). China: TPsRo. 2018 (acessado em 2018). Disponível em: http://www.stats.gov.cn/english/

26. Robinson N. Integrated traditional Chinese medicine. Complement Ther Clin Pract 2006;12:132-40.

https://doi.org/10.1016/j.ctcp.2006.01.006

27. Bhattacharyya O, Delu Y, Wong S, Bowen C. Evolution of primary care in China 1997-2009. Health Policy 2011;100:174-80. https://doi.org/10.1016/j.healthpol.2010.11.005

28. 中国. 中医药立法的相矢法律法規：参考資料. [Zhōngguó. Zhōngyīyào lìfă de xiāngguān fălû̀ făguĩ: Cānkăo zīliào. Běijīng shì: Zhōng yī guănlĩ jú bàngōngshì biān yìn]: 北京市: 中医管理局办公室编印 1998.

29. Lu A-P, Chen K-J. Chinese medicine pattern diagnosis could lead to inovation in medical sciences. Chin J Integr Med 2011;17:811-7. https://doi.org/10.1007/s11655-011-0891-z

30.Dobos G, Tao I. The model of Western integrative medicine: the role of Chinese medicine. Chin J Integr Med 2011;17:11-20. https://doi.org/10.1007/s11655-011-0601-x

31.Park $H$, Lee $H$, Shin B, Liu J, Shang Q. Traditional Chinese medicine in China, Korea, and Japan: a brief introduction and comparison. Evid Based Complement Alternant Med 2012;2012:429103. https://doi.org/10.1155/2012/429103

32. $\mathrm{Xu} \mathrm{J,} \mathrm{Yang} \mathrm{Y.} \mathrm{Traditional} \mathrm{Chinese} \mathrm{medicine} \mathrm{in} \mathrm{the} \mathrm{Chinese} \mathrm{health}$ care system. Health Policy 2009;90:133-9.

https://doi.org/10.1016/j.healthpol.2008.09.003

33. Wang J, Kushner K, Frey JJ 3rd, Ping Du X, Qian N. Primary care reform in the Peoples' Republic of China: implications for training family physicians for the world's largest country. Fam Med 2007;39:639-43.

https://fammedarchives.blob.core.windows.net/imagesandpdfs/fmhu b/fm2007/October/Jie639.pdf

34.Ren X, Yin J, Wang B, Roy Schwarz M. A descriptive analysis of medical education in China. Med Teach 2008;30:667-72. https://doi.org/10.1080/01421590802155100

35.Yang J, Guo A, Wang Y, Zhao Y, Yang X, Li H, et al. Human resource staffing and service functions of community health services organizations in China. Ann Fam Med 2008;6:421-7. https://doi.org/10.1370/afm.888 
36.Shi FD, Jia JP. Neurology and neurologic practice in China. Neurology $2011 ; 77: 1986-92$.

https://doi.org/10.1212/WNL.0b013e31823a0ed3

37.Gao PF, Watanabe K. Introduction of the World Health Organization project of the International Classification of Traditional Medicine. Zhong Xi Yi Jie He Xue Bao 2011;9:1161-4. https://doi.org/10.3736/jcim20111101

38.Wang J, Xiong X. Current situation and perspectives of clinical study in integrative medicine in china. Evid Based Complement Alternat Med 2012;2012:268542.

https://doi.org/10.1155/2012/268542

39.Jiang $M$, Zhang $C$, Zheng G, Guo $H$, Li L, Yang J, et al. Traditional chinese medicine zheng in the era of evidence-based medicine: a literature analysis. Evid Based Complement Alternat Med 2012;2012:409568. https://doi.org/10.1155/2012/409568

40. $\mathrm{Xu} \mathrm{H}$, Chen $\mathrm{KJ}$. Integrating traditional medicine with biomedicine towards a patient-centered healthcare system. Chin J Integr Med 2011;17:83-4. https://doi.org/10.1007/s11655-011-0641-2

41.Zhang D, Yin P, Freemantle N, Jordan R, Zhong N, Cheng KK. An assessment of the quality of randomised controlled trials conducted in China. Trials 2008;9:22. https://doi.org/10.1186/1745-6215-9-22

42. Nogales-Gaete J. Medicina alternativa y complementaria Complementary and alternative medicine. Rev Chil Neuro-Psiquiat 2004;42:243-50. http://dx.doi.org/10.4067/S071792272004000400001

43. Fan R. Modern western science as a standard for TCM: a critical appraisal. J Law Med Ethics 2003;31:213-21.

https://doi.org/10.1111/j.1748-720X.2003.tb00082.x

44.Fan R, Holliday I. Which medicine? Whose standard? Critical reflections on medical integration on China. J Med Ethics 2007;33:454-61. https://doi.org/10.1136/jme.2006.017483

45. Li H, Yu W. Enhancing community system in China's recent health reform: an effort to improve equity in essencial health care. Health Pol 2011;99:167-73.

https://doi.org/10.1016/j.healthpol.2010.08.006

46.Zhao XF, Su SJ, Yun G, Guo YH, Wang S. Mortality and recurrence of vascular disease among stroke patients treated with combined TCM therapy. J Tradit Chin Med 2012;32:173-8.

https://doi.org/10.1016/s0254-6272(13)60007-2

47.Gong $X$, Sucher NJ. Stroke therapy in traditional Chinese medicine (TCM): prospects for drug discovery and development. Phytomedicine 2002;9:478-84. https://doi.org/10.1078/09447110260571760

48. Li LM, Tang JL, Li J, Jiang Y, Griffiths SM. The need for integration in health sciences sets the future direction for public health education. Public Health $2011 ; 125$.

https://doi.org/10.1016/j.puhe.2010.10.008 